\title{
Biotechnische Fortpflanzungssteuerung bei Sauen und mögliche verdeckte Genfrequenzänderung für das Brunstverhalten
}

\author{
Herrn Professor Dr. Dr. h. c. Helmuth Pfeiffer zum 70. Geburtstag gewidmet
}

\begin{abstract}
Title of the paper: Biotechnical control of reproduction in sows and the opportunity of a hidden change of gene frequency for estrous behaviour

Since more than three decades hormones have been used for the synchronisation of ovulation in sows often combined with timed artificial insemination. This will be done without considering the estrous behaviour. A selection with regard to symptoms of standing behaviour is not necessary. Regarding to that it is possible to breed pigs that are not suitable for that purpose.

The aim of this study was to examine the opportunity of hidden gene frequency changes in the genetics of pigs in two herds of German Landrace. This will be shown by the example of the standing behaviour and especially the time they need to come in heat after weaning of the piglets.

The basic datas was provided by two pig-breeding farms. The calculation is based only on the information to the 2nd and 3rd litter of these sows in order to reduce the influence of selection. It is assumed that the path of heredity is monofacturial. Only pigs with the genotype "aa" will be negatively affected. At the beginning the father of boars (SS) is the sole carrier of the defect gene. The gene frequency, frequency for homozygotes and the correct number of concerned pigs after 10 generations will be calculated for a more accurate description of the expansion of this gene.

The gene frequency results in the balance of $0.4 \%$. This figure has already been achieved after eight generations. According to this value the frequency of homozygotes is $0.0017 \%$ on all paths of heredity. After ten generations the population will contain 0.0093 animals of the homozygot recessive genotype "aa". There will be 0.0055 male and 0.0038 female pigs of the negative genotype.

Finally, no negative effect of the usage of hormones for the synchronisation of ovulation in pigs has been proved.
\end{abstract}

Key Words: sows, biotechnical synchronization of ovulation, time orientated insemination, estrous behaviour, gene frequency

\section{Zusammenfassung}

In der Schweinezucht und -produktion werden seit mehr als drei Jahrzehnten Gonadotropine eingesetzt. Durch die terminorientiert durchgeführte Besamung kann die genaue Beobachtung des Duldungseintritts nach dem Absetzen in den Hintergrund treten. In diesem reglementierten System besteht die Gefahr einer verdeckten Genfrequenzänderung. Das Ziel dieser Untersuchung beinhaltet eine Kalkulation des Einflusses eines Defektgens auf zwei geschlossene Populationen der Deutschen Landrasse für den Verlauf von 10 Generationen. Das schadhafte Gen wird dabei definiert als die Ursache für einen verspäteten Duldungseintritt nach dem Absetzen. Um den Einfluss der Selektion möglichst zu reduzieren, werden nur die Angaben zum 2. und 3. Wurf berücksichtigt. Da ein monofaktorieller Erbgang modellhaft unterstellt wird, werden nur Tiere des Genotyps „aa“ negativ betroffen. Für die Berechnung wird die Population auf die vier Erbpfade, Ebervater, Ebermutter, Sauenvater und Sauenmutter, unterteilt. Zu Beginn besitzt nur ein Ebervater den Genotyp „Aa“ und ist somit Träger des defekten Gens. Für eine exakte Beschreibung des Ausbreitungsgrades des Defektgens werden die Genfrequenz, die Frequenz der Homozygoten und die genaue Anzahl betroffener Tiere ermittelt. 
Im Ergebnis beträgt die Genfrequenz im Mittel 0,4\%; die Frequenz der Homozygoten 0,0017\%. Nach 10 Generationen befinden sich in der Population 0,0093 Tiere des Genotyps „aa“. Ein negativer Einfluss durch die Gabe von Gonadotropinen hinsichtlich einer verdeckten Genfrequenzänderung ist somit nicht nachweisbar.

Schlüsselwörter: Sauen, biotechnische Ovulationssynchronisation, terminorientierte Besamung, Brunstverhalten, Genfrequenz

\section{Einleitung}

Biotechnische Verfahren zur Steuerung der Fortpflanzung werden beim weiblichen Schwein bereits seit Beginn der 70er Jahre systematisch und erfolgreich angewendet (KÖNIG und HÜHN, 1997). Als Züchtungstechnik assoziierte Verfahren fokusieren sie primär die Ovarfunktion, weil im Komplex der Fortpflanzungsmechanismen die Terminisierung der Ovulationen letztendlich die größte und weitreichendste Bedeutung für die Ferkel- und Mastschweineproduktion hat. Grundlage, Inhalt und Ziel dieser Verfahren fasste KÖNIG bereits 1973 dahin gehend zusammen, dass sie beruhend auf optimalen zootechnischen Voraussetzungen, planmäßig in die physiologischen Abläufe der Fortpflanzung bei der Sau eingreifen, um auf die Ferkelproduktion leistungssichernd, leistungssteigernd und terminlich regulierend zu wirken. Damit war die wesentliche und gleichzeitig umfassende Richtschnur für deren Anwendung in der praktischen Schweineproduktion vorgegeben.

Im Hinblick auf die Belegung von Sauen in großen Gruppen erlangten in den folgenden Jahren vor allem drei Verfahren eine maßgebliche praktische Bedeutung:

- Brunstsynchronisation bei abgesetzten Sauen mit Hilfe von eCG (PMSG),

- Brunstsynchronisation bei zuchtreifen Jungsauen und die

- Ovulationssynchronisation bei Jung- und Altsauen mit anschließender terminorientierter Besamung.

Diese Verfahren trugen wesentlich dazu bei, dass sich in der Schweineproduktion die Arbeit nach Zyklogrammen mit integrierten Serviceperioden noch fester etablierte als in den Jahren zuvor (HOFMANN, 1959; KÖNIG, 1973). Besonders in den Zweigen der Tierproduktion mit kürzeren Produktions- und Reproduktionszyklen, wie die Ferkelproduktion, erweist sich die terminliche Ausrichtung des Produktionsmanagements nach diesen Gesichtspunkten aus wirtschaftlichen und tiergesundheitlichen Aspekten als äußerst notwendig (KÖNIG und HÜHN, 1997).

Heute, bei immer kürzeren Produktionszeiten, setzt ein derartiges System das gleichzeitige Eintreten der Brunst bei den Sauen einer Gruppe voraus. Das erfordert die exogene Steuerung des individuellen Östrus, besser noch der individuellen Ovulation mithilfe gonadotrop wirkender Hormone wie eCG (PMSG) und hCG sowie GnRH.

Das biotechnische Verfahren der Brunststimulation bei Altsauen hat zum Ziel nach dem Absetzen der Ferkel Eintritt, Dauer und Intensität der Brunst im Hinblick auf die nach Besamung zu erzielenden Trächtigkeitsrate und Wurfgröße im physiologischen Maß zu sichern (HÜHN, 1995), weil nur so mit hoher Wahrscheinlichkeit auf eine Ovulation geschlossen werden kann (CLAUS et al., 1993). Bei zuchtreifen Jungsauen bewirkt das Verfahren der Brunstsynchronisation die relative Gleichschaltung der Östren (WÄHNER, 2002). Die Festlegung des Besamungstermins erfolgt in beiden Fällen auf der Grundlage der Ergebnisse aus der Brunstdiagnose (WEITZE et al., 1994). 
Generell wird damit dem berechtigten Anliegen einer terminisierten Ferkelproduktion jedoch nur bedingt entsprochen, weil zwar die Östren mit ihren hohen individuellen Varianzen für Eintritt und Dauer (SCHLEGEL und SKLENAR, 1973; HENZE et al., 1986; WABERSKI und WEITZE, 1996; SOEDE und KEMP, 1997), jedoch kaum die Ovulationen synchronisiert werden. Die Heritabilitätskoeffizienten für diese Merkmale sind folglich sehr niedrig (RITTER und SCHAAF, 1978). All das erschwert zweifellos die Festlegung des optimalen Besamungstermins (HENZE und KRETZSCHMAR, 1980; HENZE et al., 1982; WEITZE et al., 1994) und steht der Grundvoraussetzung für die terminisierte Ferkelproduktion, nämlich die gleichzeitig durchzuführende Besamung aller Sauen einer Gruppe, prinzipiell entgegen.

Hier liegt der Beweggrund für die Entwicklung des biotechnischen Verfahrens der Ovulationssynchronisation mit terminorientierter Besamung bei Jung- und abgesetzten Altsauen (HÜHN et al., 1996; WÄHNER, 2002). Es baut auf dem Verfahren der Brunstsynchronisation auf und enthält den zusätzlichen Behandlungsschritt der hormonellen Ovulationsstimulation mit Hilfe von hCG (HÜHN et al., 1976; SCHLEGEL et al. 1989) oder GnRH (BRÜSSOW, 1978; WÄHNER, 1989, 2002; WÄHNER und HÜHN, 1996; BRÜSSOW et al., 1993). Die damit erreichte, relative Gleichschaltung der Ovulationen innerhalb der Östren ermöglicht die Besamungen zeitorientiert vorzunehmen, und primär damit verbundene produktionsorganisatorische Vorteile zu nutzen. Der leistungssteigernde Effekt wirkt sich vor allem darin aus, dass von den zur Besamung aufgestellten Sauen nahezu keine Tiere wegen fehlender Duldung ausfallen. Im Vergleich zur duldungsorientierten Besamung sind demnach 5 bis $10 \%$ weniger Sauen erforderlich, um die gleiche Anzahl an Würfen zu erhalten. Grundsätzlich ist auch bei diesem Verfahren die Ausprägung des Östrus zu den feststehenden Besamungsterminen für ein hohes Befruchtungsergebnis (WÄHNER et al., 1998; HEINZE und SASSMANN, 2001) förderlich und notwendig.

Bei Anwendung der terminorientierten Besamung kann jedoch die intensive und über den gesamten Brunstzeitraum durchzuführende Kontrolle des Östrus in den Hintergrund treten bzw. sogar wegfallen. Letzteres war vor allem im Zeitraum von Beginn der 70er bis Mitte der 80er Jahre als Vorteil gegenüber anderen Fortpflanzungsverfahren betont worden (SCHREMMER, 1975; HÜHN et al., 1976; SCHLEGEL, 1984). Es bestand und besteht folglich die theoretische Möglichkeit, dass Sauen, die zu einem verzögerten Duldungseintritt neigen, bei konsequenter Anwendung der biotechnischen Ovulationssynchronisation und terminorientierten Besamung unentdeckt in der Herde verbleiben. Werden diese dann als Zuchtsauen eingesetzt, könnte sich ein schädigendes Gen unbemerkt in der Herde manifestieren und folglich zu einer verdeckten Genfrequenzänderung mit möglicherweise beeinträchtigten Merkmalsausprägungen, in diesem Falle des Brunstverhaltens, führen.

Das Wissen um diese Problematik fand von Anbeginn an seine Berücksichtigung in den Hinweisen für die Anwendung biotechnischer Verfahren der Fortpflanzung in den Schweinezuchtbetrieben. Das Verfahren der Ovulationssynchronisation empfahl sich insofern zunächst nur für die Stufe der Mastferkelerzeugung, weniger für die zuchtaktiven Ebenen (KÖNIG, 1982). Desweiteren sind züchtungsbiologische Untersuchungen bei Schweinen zu den Auswirkungen der fortgesetzten Anwendung, speziell der Ovulationssynchronisation, in der Generationsfolge zur Begleitung einer risikolosen landesweiten praktischen Handhabung in den Sauenbeständen notwendig, um gegebe- 
nenfalls notwendige Maßnahmen zur Abwehr einer möglichen Gefahr ergreifen zu können. Diesem Anliegen dient die hier vorzustellende Untersuchung.

\section{Zielstellung}

Bisher durchgeführte Untersuchungen von SIMIANER (1993) sowie KLAUTSCHEK und KÖNIG (1994) konnten generell die theoretische Möglichkeit einer verdeckten Genfrequenzänderung anhand eines kalkulatorischen Modells bestätigen. Es lagen jedoch die ermittelten Zahlenwerte für betroffene Tiere so niedrig, dass ein nachhaltiger Einfluss auf die Population ausgeschlossen werden konnte. Die Berechnungen der genannten Autoren beruhen dabei ausschließlich auf hypothetischen Zahlenwerten einer schematisierten Population. In den vorliegenden Untersuchungen sollten mögliche Genfrequenzänderung für ein Defektgen nun im Rahmen einer Praxisuntersuchung analysiert werden. Es wurde dafür ein Defektgen mit seinen Auswirkungen auf das Brunstverhalten mit dem Merkmal "Duldungseintritt nach dem Absetzen der Ferkel" gewählt. Davon betroffene Sauen würden zu einem verspäteten Duldungseintritt neigen oder generell keine Brunstsymptome aufzeigen.

\section{Material und Methoden}

\section{Datenerhebung}

Für die Untersuchung wurden zwei Betriebe mit Sauen der Deutschen Landrasse ausgewählt, in denen die Dokumentation bezüglich des Brunstverhaltens zur Besamung sehr exakt vorgenommen und archiviert wurde. Im Hinblick auf die biotechnische Behandlung der Altsauen sind beide Unternehmen mit einander vergleichbar. Alle Sauen wurden dem biotechnischen Fortpflanzungsverfahren der Ovulationssynchronisation unterzogen. Dazu erhielten die Sauen nach dem Absetzen der Ferkel 800 IE eCG und nach weiteren 73 Stunden 500 IE hCG. Trotz terminorientiert durchgeführter Besamung wurde über den gesamten Erfassungszeitraum zur Besamung eine zweimal tägliche Brunstkontrolle durchgeführt.

Die aufgenommenen Daten umfassen einen Zeitraum von 13 Jahren. Um den Einfluss der Selektion möglichst gering zu halten, finden nur die Angaben zum zweiten und dritten Wurf Berücksichtigung. So wird vermieden, dass Sauen mit einer sehr guten Fruchtbarkeit und hohen Wurfzahlen das Ergebnis verfälschen könnten.

Die Auswahl der Daten erfolgte zufällig. Auf diese Weise soll der Stichprobencharakter gewahrt bleiben. Für die Berechnung der Genfrequenzen, der Frequenz der Homozygoten und deren genauen Anzahlen wird die Datenmenge beider Betriebe als Grundgesamtheit behandelt.

Es ist zu beachten, dass die Erfassung der Daten rekursiv zu den derzeit vorhandenen Beständen erfolgte. Die Ausgangsbasis bilden die jüngsten, in der Zucht integrierten, Sauen, die mindestens drei Würfe erbracht haben sollten. Auf diese Weise wurden „Familien“ bis in die 6. Generation zurückverfolgt. Ebenso war es möglich verwandtschaftliche Beziehungen zwischen den „Familien“ aufzuzeigen.

\section{Doppelung}

Die aufgenommenen Daten setzen sich aus den Werten mehrerer Familien zusammen. Weil die jeweiligen Sauen stichprobenartig und willkürlich ausgewählt wurden, kommt es vor, dass einige Familien zum Teil einen gleichen Stammbaum aufweisen. Für die anschließenden Berechnungen war Folgendes zu beachten: Einige Tiere üben 
offensichtlich einen größeren genetischen Einfluss auf die Population aus als die Tiere, die in der Stichprobe nur einmal Verwendung finden. Da das angewandte Rechenschema lediglich auf der Anzahl der Tiere aufbaut, aber die Häufigkeit ihres Vorkommens unberücksichtigt lässt, werden alle Berechnungen unter Beachtung dieser Tatsache in zwei Grundvarianten durchgeführt.

- Variante I: Das mehrfache Auftreten einiger Sauen und Eber wird berücksichtigt.

- Variante II: Jedes Tier wird nur einfach gewertet.

Demnach umschreibt Variante I die Zusammensetzung der untersuchten Population besser als Variante II.

\section{Berechnung}

Die Untersuchung erfolgt auf der Grundlage des Rechenmodells von KLAUTSCHEK und KÖNIG (1994). Daher wird, obwohl das Brunstverhalten ein polyfaktorielles System darstellt, modellhaft ein monofaktorieller Erbgang zu Grunde gelegt. Eine Beeinträchtigung der Tiere wird nur in der homozygot rezessiven Form „aa“ sichtbar. Als Anlagenträger werden die Eberväter definiert. Für eine möglichst fundierte Aussage hinsichtlich der Verbreitung eines derartigen Defektgens innerhalb einer geschlossenen Population werden verschiedene Häufigkeiten des Auftretens simuliert. Die Kalkulation erfolgt deshalb in 4 Szenarien mit unterschiedlichen Schädigungsstufen. In Szenario 1 ist lediglich ein Ebervater heterozygot veranlagt, in Szenario 2 zwei Eberväter, in Szenario 3 fünf und in Szenario 4, dem schlimmsten aller anzunehmenden Fälle, sind alle Eberväter der Ausgangspopulation Träger des Defektgens. Die vier Szenarien wurden in den beiden genannten Varianten geprüft.

Nach der Auswertung der Pedigreedaten beider Betriebe bilden die Werte von 552 Tieren die Kalkulationsgrundlage für Variante I. In Variante II wurden die Werte von 292 Tieren berechnet. Dabei erfolgt die Aufteilung der Gesamtanzahl hinsichtlich der vier Erbpfade (Ebervater, Sauenvater, Ebermutter und Sauenmutter) sowie der Verteilung in Bezug auf die Anpaarungsmöglichkeiten in Anlehnung an das bei KLAUTSCHEK und KÖNIG (1994) enthaltene Modell (Tab. 1).

Tabelle 1

Anzahl Nachkommen auf den Zuchtpfaden „Variante I“ (Number of descendants related to the path of heredity "variant I")

\begin{tabular}{|c|c|c|c|c|c|c|}
\hline \multirow{2}{*}{$\begin{array}{l}\text { Paarung } \\
E V \times E M\end{array}$} & $\begin{array}{c}\text { Vater-Sohn } \\
\text { Ebervater (EV) }\end{array}$ & \multicolumn{2}{|c|}{$\begin{array}{c}\text { Vater-Tochter } \\
\text { Sauenvater (SV) }\end{array}$} & \multicolumn{2}{|c|}{$\begin{array}{c}\text { Mutter-Sohn } \\
\text { Ebermutter (EM) }\end{array}$} & $\begin{array}{c}\text { Mutter-Tochter } \\
\text { Sauenmutter (SM) }\end{array}$ \\
\hline & $45 \quad$ (A) & 28 & (B) & 2 & (C) & $0 \quad$ (D) \\
\hline$E V \times S M$ & & 42 & (E) & 0,5 & (F) & $26,3 \quad(G)$ \\
\hline$S V \times E M$ & & 210 & $(\mathrm{H})$ & 0,5 & (I) & $13,2 \quad(\mathrm{~J})$ \\
\hline$S V \times S M$ & & & & 0 & $(\mathrm{~K})$ & $184,5 \quad(\mathrm{P})$ \\
\hline Gesamt & $45 \quad(L)$ & 280 & (M) & 3 & $(\mathrm{~N})$ & 224 (O) \\
\hline
\end{tabular}

Die zwischen beiden Varianten unterschiedliche Gesamtanzahl der Tiere spiegelt sich ebenfalls in ihrer Verteilung auf die Erbpfade wider (Tab. 2). 
Tabelle 2

Anzahl Tiere auf den Erbpfaden in Variante I und II (Number of animals on their path of heredity "variant I and II")

\begin{tabular}{cccccc}
\hline Erbpfad & Ebervater & Sauenvater & Ebermutter & Sauenmutter & gesamt \\
\hline Variante I & 45 & 280 & 3 & 224 & 552 \\
Variante II & 18 & 103 & 3 & 168 & 292 \\
\hline
\end{tabular}

$\mathrm{Zu}$ Beginn wird die Genfrequenz berechnet, im Anschluss daran die Frequenz der Homozygoten. Daran anknüpfend kann die letztendliche Anzahl betroffener Tiere abgeschätzt werden. Dabei werden die Ergebnisse für den Verlauf von 10 Generationen simuliert.

Die Berechnung der Genfrequenz der ersten Generation erfolgt nach dem in Tabelle 3 dargestellten Muster.

Tabelle 3

Gleichungsübersicht zur Berechnung der Genfrequenz der ersten Generation (Calculation of gene frequency of the $1^{\text {st }}$ generation)

\begin{tabular}{|c|c|c|c|c|}
\hline Pfad & & Gle & & \\
\hline \multirow{3}{*}{ EV } & \multirow{3}{*}{$\mathrm{q}_{\mathrm{EV}}^{1}=$} & Zahl Defektallele & \multirow{2}{*}{+} & \multirow{2}{*}{$\mathrm{Bx} \mathrm{q}^{0} \mathrm{sv}$} \\
\hline & & $2 \times A$ & & \\
\hline & & & 2 & \\
\hline \multirow[t]{2}{*}{ SV } & \multirow{2}{*}{$\mathrm{q}_{\mathrm{sv}}^{1}=$} & $(\mathrm{B}+\mathrm{E}) \mathrm{xq}_{\mathrm{EV}}^{1}$ & + & $\mathrm{Hxq} \mathrm{q}_{\mathrm{sV}}$ \\
\hline & & & M & \\
\hline \multirow[t]{2}{*}{ EM } & \multirow{2}{*}{$\mathrm{q}_{\mathrm{EM}}^{1}=$} & $(\mathrm{C}+\mathrm{F}) \mathrm{xq}_{\mathrm{EV}}^{1}$ & + & $(I+K) \times q_{s v}^{0}$ \\
\hline & & & $\mathrm{N}$ & \\
\hline \multirow[t]{2}{*}{ SM } & \multirow[t]{2}{*}{$\mathrm{q}_{\mathrm{SM}}^{1}=$} & $(\mathrm{D}+\mathrm{G}) \times \mathrm{q}_{\mathrm{EV}}^{1}$ & + & $(\mathrm{J}+\mathrm{P}) \mathrm{xq}_{\mathrm{sv}}^{0}$ \\
\hline & & & $\mathrm{O}$ & \\
\hline
\end{tabular}

\section{Ergebnisse}

In Abbildung 1 ist ein Überblick zum Duldungsgeschehen in beiden untersuchten Betrieben enthalten.

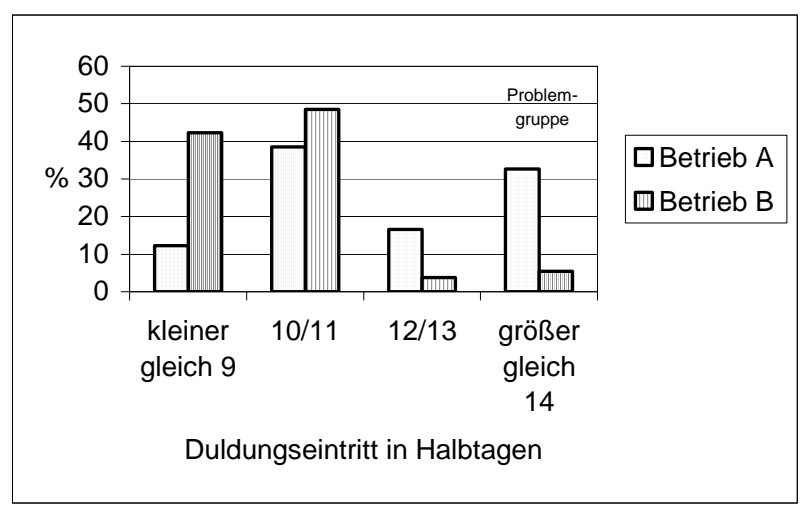

Abb. 1: Überblick zum Duldungseintritt in beiden Untersuchungsbetrieben (Overview of the situation on the pig breeding farms according to standing behaviour) 
Demnach liegen bei den Sauen beider Bestände im Duldungseintritt große Unterschiede vor.

Die im Folgenden aufgezeigten Ergebnisse beziehen sich aus Gründen der Übersichtlichkeit auf Variante I / Szenario 1.

Unter Anwendung des in Tabelle 3 abgebildeten Gleichungsweges lassen sich die Genfrequenzen für alle vier Erbpfade rekursiv für 10 Generationen kalkulieren. Graphisch dargestellt, ergibt sich für die Entwicklung der Genfrequenz in Variante I / Szenario 1 (ein Ebervater als Merkmalsträger) die in Abbildung 2 vorgelegte Tendenz.

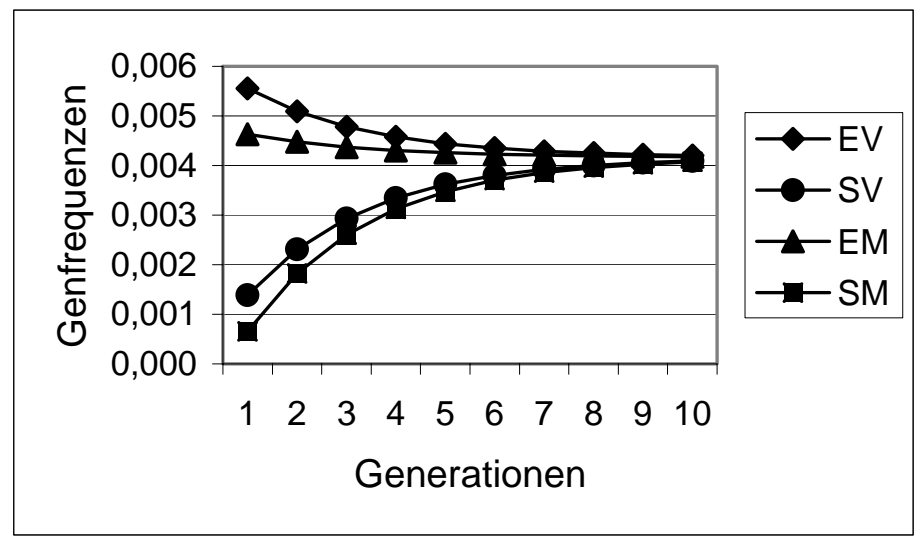

Abb. 2: Entwicklung der Genfrequenz (Variante I / Szenario 1) für den Verlauf von 10 Generationen (Gene frequency on path of heredity in the course of 10 generations $-1^{\text {st }}$ variant $/ 1^{\text {st }}$ scenery)

Abgeleitet aus der Genfrequenz, diese erhoben zum Quadrat, wird die Frequenz der Homozygoten ermittelt. Daher ergibt sich (ebenfalls Variante I / Szenario 1) die in Abbildung 3 aufgezeigte graphische Darstellung.

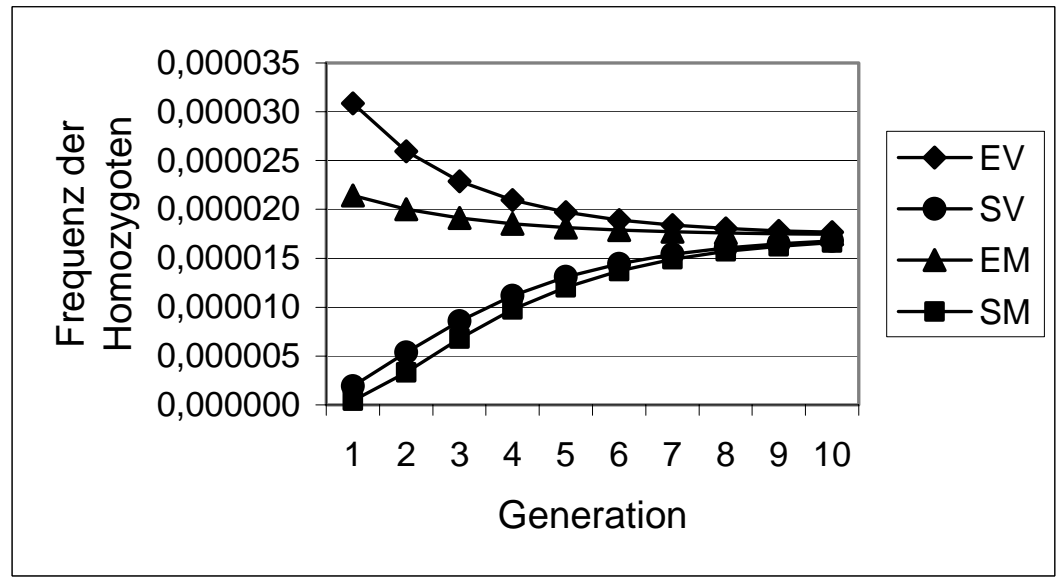

Abb. 3: Entwicklung der Frequenz der Homozygoten (Variante I / Szenario 1) für den Verlauf von 10 Generationen (Frequency of homozygotes on path of heredity in the course of 10 generations - $1^{\text {st }}$ variant / $1^{\text {st }}$ scenery)

Sowohl für die Genfrequenz als auch für die Frequenz der Homozygoten ist ein ähnlicher Verlauf zu verzeichnen. Nach jeweils 8 Generationen bildet sich eine Gleichgewichtsfrequenz, die über die 10. Generation hinaus Bestand hat.

Die letztendliche Anzahl homozygot rezessiver Tiere ergibt sich als Produkt aus Homozygotenfrequenz der entsprechenden Generation und Erbpfad und der jeweiligen Gesamtanzahl der Tiere aus dem Ausgangstableau. Für Variante I / Szenario 1 ergeben 
sich in der Summe 0,0093 geschädigte Tiere nach 10 Generationen. Dabei entfallen 0,0055 auf männliche und 0,0038 auf weibliche Zuchttiere. Zum Vergleich der vier Szenarien in Variante I sind die Ergebnisse in Tabelle 4 gegenübergestellt.

Tabelle 4

Vergleich der Ergebnisse für alle 4 geprüften Szenarien in Variante I (Comparison of the results of all proved sceneries in the $1^{\text {st }}$ variant)

\begin{tabular}{ccccc}
\hline Szenario & 1 & 2 & 3 & 4 \\
\cline { 2 - 5 } & 1 Eber Aa & 2 Eber Aa & 5 Eber Aa & alle Eber Aa \\
\hline Genfrequenz & 0,004 & 0,008 & 0,021 & 0,186 \\
Frequenz der Homozygoten & 0,000017 & 0,000068 & 0,00043 & 0,034 \\
Anzahl betroffener Tiere insgesamt & 0,0093 & 0,037 & 0,232 & 18,75 \\
davon weiblich & 0,0038 & 0,015 & 0,107 & 7,64 \\
\hline
\end{tabular}

Da das Untersuchungsmerkmal „Duldungseintritt nach dem Absetzen“ nur bei weiblichen Tieren sichtbar wird, ist die Anzahl betroffener Zuchtsauen relevant. Die Entwicklung der Anzahl homozygot rezessiver Sauen ist in Tabelle 5 für beide Varianten in allen geprüften Szenarien dargestellt.

Tabelle 5

Anzahl geschädigter Zuchtsauen nach 10 Generationen in den Varianten I und II in den Szenarien 1 - 4 ( Number of concerned sows after 10 generations for both variants and all sceneries)

\begin{tabular}{ccccc}
\hline Szenario & 1 & 2 & 3 & 4 \\
\hline Variante I & 0,004 & 0,015 & 0,107 & 7,640 \\
Variante II & 0,018 & 0,071 & 0,444 & 5,755 \\
\hline
\end{tabular}

Lediglich in Szenario 4 ist in beiden Varianten mehr als eine Zuchtsau von der genetischen Schädigung betroffen. Unabhängig von der untersuchten Variante bleiben die Werte in den Szenarien 1 - 3 kleiner als 0,5.

\section{Diskussion}

Bei abgesetzten und anschließend biotechnisch ovulationssynchronisierten Sauen ist das Merkmal „Brunsteintritt nach dem Absetzen“ in besonderer Weise wissenschaftlich interessant, weil es im Rahmen der terminorientierten Besamung nur in zweiter Hinsicht bedeutungsvoll zu sein scheint. Insofern war es aufschlussreich zu prüfen, ob nach 10 Generationen kontinuierlicher Anwendung dieses biotechnischen Verfahrens bei Sauen gleichbleibend durchgehend geführter Herden hier möglicherweise Veränderungen sich ergeben haben könnten. Dazu wurden zwei Bestände der Deutschen Landrasse (Betriebe A und B) ausgewählt.

Generell sind für das Merkmal „Duldungseintritt nach dem Absetzen“ zwischen den Sauen der beiden untersuchten Unternehmen große Unterschiede festzustellen. Bereits HENZE et al. (1986) weisen auf derartige Varianzen hin. In Betrieb A gelangen über 30 \% aller Sauen nur verspätet in die Brunst (Problemgruppe), während in Betrieb B das Duldungsgeschehen wesentlich konzentrierter verläuft; bei über 90 \% aller Sauen sind nach 10 bis 11 Halbtagen Duldungsmerkmale zu verzeichnen.

Im Regelfall zeigen Altsauen 5 bis $51 / 2$ Tage nach dem Absetzen erste Brunstmerkmale (WEITZE et al., 1994; HÜHN et al., 1996). Mit dem hier angewandten Re- 
chenmodell wurde daher überprüft, ob der hohe Anteil Sauen in der Problemgruppe des Betriebes A auf eine verdeckte Genfrequenzänderung zurückzuführen ist oder ob hier betriebsspezifische Gründe vorliegen.

Die Kalkulation der Genfrequenz weist nach 10 Generationen eine Gleichgewichtsfrequenz von 0,004 auf. Diese hat Bestand auch über die 10. Generation hinaus. Auf dem Pfad der Eberväter (EV) ist unabhängig von der geprüften Variante und Szenario der höchste Ausgangswert zu verzeichnen, auf dem Pfad der Sauenmütter (SM) der niedrigste.

Aufgrund des Rechenweges nach SIMIANER (1993) bzw. KLAUTSCHEK und KÖNIG (1994) ist die Entwicklung der Homozygotenfrequenz vergleichbar mit der der Genfrequenz. Wiederum weisen die Eberväter (EV) den höchsten Anfangswert auf, die Sauenmütter (SM) den niedrigsten. Nach acht Generationen bildet sich die Gleichgewichtsfrequenz bei 0,000017 heraus.

Die Anzahl homozygot rezessiver Tiere nach 10 Generationen ist das entscheidende Kriterium zur Abschätzung des Einflusses und des Verbreitungsgrades eines Defektgens in der Population. Die ermittelten Anzahlen homozygot rezessiver Tiere nach 10 Generationen sind sehr gering, kleiner als 1. Werden ausschließlich die Zuchtsauen bewertet, sind weniger als 0,5 Tiere geschädigt bzw. kommen verspätet in die Brunst oder weisen generell keine Duldungsmerkmale auf. Die geprüften Szenarien sind hinsichtlich ihrer Wahrscheinlichkeit differenziert zu betrachten. Dem Szenario 4, in dem angenommen wurde, dass alle eingesetzten Eberväter heterozygot und deshalb Anlagenträger für das Defektgen sind, ist demzufolge die geringste Bedeutung beizumessen und wird somit in der abschließenden Betrachtung nicht berücksichtigt.

Wegen dieser niedrigen Zahlenwerte und auf Grund der Tatsache, dass die in der züchterischen Praxis übliche Selektion nicht berücksichtigt wurde, kann ein negativer Einfluss von Hormonen zur Zyklussteuerung auf das Merkmal „Duldungseintritt nach dem Absetzen“ verneint werden. Der hohe Anteil an Sauen in der „Problemgruppe“ in Betrieb A ist somit nicht mit einer verdeckten Genfrequenzänderung zu erklären. Die Ursachen hierfür müssen im betrieblichen Umfeld gefunden werden.

\section{Literatur}

BRÜSSOW, K. P.:

Untersuchungen zur Erprobung eines synthetischen Gonadotropin-Releasinghormons (Gn-RH) für die biotechnische Anwendung beim weiblichen Jungschwein. Diss., AdL Berlin, 1978

BRÜSSOW, K. P.; RATKY, J.; KANITZ, E.:

The influence of exogenous GnRH on the time of ovulation in gilts - an endocrine and laparascopic study. Arch. Tierz., Dummerstorf 36 (1993), 193 - 203

CLAUS, R.; KOEK, G.; WEILER, U.:

Wie „funktioniert“ die Fruchtbarkeit? Schweinezucht und Schweinemast 41 (1993) 6, 40 - 41

HENZE, A.; KRETZSCHMAR, A.:

Zur Organisation der duldungsorientierten Besamung von Sauen. Tierzucht 34 (1980), 35 - 38

HENZE, A.; PETER, W.; KEIL, W.; TRÄBER, H.; NESTLER, W.; DINGELDEIN, J.; SCHMIDT, H.G.; v. d. MÜLBE, D.:

Künstliche Besamung Schwein. Broschüre VVB Tierzucht, Paretz, 1986

HOFMANN, F.:

Planmäßige Schweineerzeugung mit Hilfe des periodischen Abferkelsystems. Wiss. Zeitschr. d. Friedr. - Schiller-Univers., Jena, Math.-Nat. Reihe 9, 1959, H. 1/2

HÜHN, U.; SCHLEGEL, W.; KÖNIG, I.; BERGFELD, J.: Ovulationssynchronisation und terminorientierte Besamung bei Sauen. 2. verbesserte Auflage. agra buch, Markkleeberg, 1976

HÜHN, U.: PMSG - Zyklusstart bei abgesetzten Sauen. Schweinezucht und Schweinemast 43 (1995) 6, 14 - 16 
HÜHN, U.; JÖCHLE, W.; BRÜSSOW, K.P.:

Techniques developed for the control of estrus, ovulation and parturition in the East German pig industry: A review. Theriogenology 46 (1996), 911 - 924

KLAUTSCHEK, I.; KÖNIG, I.:

Biotechnische Fortpflanzungssteuerung und mögliche verdeckt Genfrequenzänderung für das Pubertätsalter. Arch. Tierz., Dummerstorf 37 (1994) 3, 301 - 308

KÖNIG, I.:

Biotechnik der Fortpflanzung in der industriemäßigen Schweineproduktion. Tierzucht 27 (1973), 234

KÖNIG, I.:

Fortpflanzung bei Schweinen. 1. Auflage; VEB Deutscher Landwirtschaftsverlag: Berlin, 1982

KÖNIG, I.; HÜHN, U.:

Zur Steuerung der Fortpflanzung bei Sauen - Eine Retrospektive. Arch. Tierz., Dummerstorf 40 (1997) 3, 239 - 256

RITTER, E.; SCHAAF, A.:

Genetische Aspekte der Fruchtbarkeit beim Schwein. Fortschrittsbericht, AdL, Berlin 1978

SCHLEGEL, W.; SKLENAR, V.:

Untersuchungen zur Brunstfeststellung und zum Brunstverlauf sowie deren Beziehungen zur Fruchtbarkeitsleistung bei Sauen. Tierzucht 27 (1973), 7 - 9

SCHLEGEL, W.:

Reproduktion des Schweinebestandes. In: PFEIFFER, H.: Schweinezucht. 3. überarbeitete Auflage; VEB Deutscher Landwirtschaftsverlag: Berlin, 1984

SCHLEGEL, W.; WÄHNER, M.; GLEI, M.

Untersuchungen zur Dosierung von Gonavet "Berlin-Chemie" zur Ovulationsstimulation im Rahmen der Ovulationssynchronisation von Jungsauen. Mh. Vet. Med. 44 (1989), 320 - 321

SCHREMMER, H. (Herausgeber): Industriemäßige Schweineproduktion. 1. Auflage, VEB Deutscher Landwirtschaftsverlag, Berlin, 1975

SIMIANER, H.:

Genfluss von Letalgenen in Rinderpopulationen. Vortrag, Biometrisches Kolloquium der Deutschen Region der Internationalen Biometrischen Gesellschaft - AG Populationsgenetik, 16. - 19.03.1993, Berlin, 1 - 15

SOEDE, N.; KEMP, B.:

Oestrus expression and timing of ovulation in pigs. $5^{\text {th }}$ Intern. Conference on Pig Reproduction, Kerkrade (NL) 1997, abstr. 41

WABERSKI, D.; WEITZE, K. F.:

Correct Timing of artificial Insemination in Pigs. Reprod. Dom. Anim. 31 (1996), 527 - 530

WÄHNER, M.:

Untersuchungen zum Einsatz eines analogen Gonadotropin-Releasinghormons zur Ovulationsstimulation bei Sauen - Ein Beitrag zur Anwendung von Gonavet ${ }^{\circledR}$ „Berlin-Chemie“ im Rahmen des biotechnischen Fortpflanzungsverfahrens der Ovulationssynchronisation. Habil.-schr. Univ. Leipzig, 1989

WÄHNER, M.; HÜHN, U.:

New Aspects of the Management of Reproduction in Pig. Reprod. Dom. Anim. 31 (1996), 477 - 482

WÄHNER, M.:

Synchronisation von Zyklus und Ovulation beim Schwein. Tierärztl. Prax. (2002), 30 (G) 252 - 260

WEITZE, K. F.; WAGNER - RIETSCHEL, H.; WABERSKI, D.; RICHTER, L.; KRIETER, J.:

The onset of heat after weaning, heat duration and ovulation as major factors in AI timing in sows. Reprod. Dom. Anim. 29 (1994), 433 - 443

Eingegangen: 01.10.2003

Akzeptiert: 23.10.2003

Anschrift der Verfasser

Dipl.-Ing. (FH) KATHLEEN FISCHER, Prof. Dr. habil. MARTIN WÄHNER

Hochschule Anhalt (FH)

Fachbereich Landwirtschaft, Ökotrophologie, Landespflege

Strenzfelder Allee 28

D-06406 Bernburg 